\title{
Microscopic Invasion Patterns and Epithelial Cell-phenotypes in Early Gastric Cancer with Submucosal Invasion
}

Kazuhiro Fukatsu ${ }^{1}$, Jun Kato ${ }^{1 *}$, Toru Niwa ${ }^{1}$, Mikitaka Iguchi ${ }^{1}$, Yousuke Muraki ${ }^{1}$, Takao Maekita ${ }^{1}$, Izumi Inoue $^{1}$, Hideyuki Tamai ${ }^{1}$, Hisanobu Deguchi $^{1}$, Kosaku Moribata $^{1}$, Yoshimasa Maeda ${ }^{1}$, Yasushi Nakamura ${ }^{2}$, Shinichi Murata ${ }^{2}$ and Masao Ichinose ${ }^{1}$

${ }^{1}$ Second Department of Internal Medicine, Wakayama Medical University, Wakayama, Japan

${ }^{2}$ Department of Pathology, Wakayama Medical University, Wakayama, Japan

"Corresponding author: Jun Kato, Second Department of Internal Medicine, Wakayama Medical University, 811-1 Kimiidera, Wakayama city, Wakayama 641-0012, Japan, Tel: +8173-441-0627; Fax: +8173-445-3616; E-mail: katojun@wakayama-med.ac.jp

Received date: Sep 04, 2015; Accepted date: Oct 31, 2015; Published date: Nov 02, 2015

Copyright: (C) 2015 Fukatsu K, et al. This is an open-access article distributed under the terms of the Creative Commons Attribution License, which permits unrestricted use, distribution, and reproduction in any medium, provided the original author and source are credited.

\begin{abstract}
Background and study aims: Early gastric cancers show gastric and/or intestinal phenotypes with specific mucin production profiles, and the phenotypes can vary with tumor progression. The aim of this study was to evaluate the correlation between tumor invasion patterns and phenotypes in the mucosa and submucosa of early gastric cancers.
\end{abstract}

Methods: Phenotypic expressions of 44 endoscopically resected gastric cancers with submucosal invasion were evaluated immunohistochemically using MUC5AC and MUC6 as gastric and MUC2 and CD10 as intestinal phenotypic markers.

Results: Cancers were classified into two patterns by invasion pattern: 19 collapsing pattern (C-pattern) tumors had cancer cells that invaded to the submucosa with expansive destruction of the muscularis mucosae, while 25 passing-through pattern (P-pattern) tumors formed focal cancer cell aggregations in the submucosa without massive destruction of the muscularis mucosae. Cancers with C-pattern invasion were likely to show similar phenotypes between the mucosa and submucosa, while phenotypes of cancers with P-pattern invasion were likely to differ between the two layers (rate of the same phenotypes: C-pattern 68\% vs. P-pattern $28 \%, p=0.008$ ). Of 22 cancers with P-pattern invasion that included the intestinal phenotype component in the mucosa, 13 (59\%) expressed the gastric phenotype alone in the submucosa.

Conclusions: Phenotype presentation in the mucosa and submucosa differ by the invasion pattern in early gastric cancer. Tumors with P-pattern invasion are likely to express the gastric phenotype in the submucosa, regardless of phenotype in the mucosa, suggesting that such cancers might achieve submucosal invasion prior to intestinalization occurring in the mucosa.

Keywords: Submucosal gastric cancer; Invasion pattern; Gastric phenotype; Intestinal phenotype

\section{Abbreviations: \\ HE: Hematoxylin and eosin; H. pylori: Helicobacter pylori}

\section{Introduction}

Gastric cancer is one of the leading causes of cancer-related deaths in Japan. With 49,129 deaths in Japan attributed to this cancer in 2012, gastric cancer remains a major health problem [1]. Recently, early gastric cancers, including mucosal and submucosal cancers, have been more frequently found due to progress in endoscopic modalities, and endoscopic resection has been widely performed. Because approximately $20 \%$ of gastric cancers with submucosal invasion have lymph node metastases [2], endoscopic resection for these lesions should be judiciously applied. In this context, the clinical processes and basic mechanisms of cancer infiltration into the submucosa appear to be relevant.
It is known empirically that gastric cancer invades to the submucosal layer in either of the following two manners, as reported approximately 50 years ago [3,4]: first, tumor cells destroy the muscularis mucosae widely and infiltrate diffusely into the submucosa; and second, tumor cells pass through the muscularis mucosae without destroying it and form focal aggregations in the submucosa. However, the clinical and basic differences between these two types of manners of submucosal invasion are largely unknown. Knowing what accounts for the difference in the manner of invasion may facilitate the elucidation of mechanisms of submucosal invasion of gastric cancer, and it may become useful in determining the indication for endoscopic resection.

Lauren et al. classified human gastric cancers into two major groups, the 'intestinal' and 'diffuse' types [5], which are considered to closely correspond to the 'differentiated' and 'undifferentiated' types, respectively, of Nakamura et al. [6]. In this context, gastric and intestinal phenotypes have been proposed, based on the immunohistochemistry for specific mucin production of gastric cancer cells. It has been considered that differentiated gastric carcinoma arises from atrophic mucosa or intestinal metaplasia, expressing intestinal mucin phenotypes, and that undifferentiated gastric carcinoma arises 
from non-atrophic gastric mucosa of young persons or women expressing gastric mucin phenotypes [5-8]. However, previous immunohistochemical studies have proven that differentiated gastric cancer usually expresses the gastric 'not intestinal' phenotype in its very early stage, and thereafter, the shift to the intestinal phenotype occurs with cancer progression [9-15]. However, this type of phenotype change has been reported in early gastric cancer confined to the mucosal layer. Few reports indicated the phenotype difference of cancer cells between the mucosal and submucosal layers.

In this study, therefore, the phenotype difference between the mucosa and submucosa was examined in early gastric cancer with submucosal invasion. In particular, the correlation of invasion patterns of cancer cells with the phenotype changes was specifically determined. The present investigation may partly help elucidate the mechanisms of cancer invasion into the submucosa of gastric cancer.

\section{Materials and Methods}

\section{Samples and tissue collection}

Clinical samples were collected from 809 primary solitary gastric cancer specimens that had been resected endoscopically at Wakayama University Hospital between May 2002 and June 2013. The criteria for the collection of samples were early differentiated gastric cancer (tub1, tub2, or pap, according to the Japanese Classification of Gastric Carcinoma [16]) with submucosal invasion, and they were appropriate for histological analysis. There were 52 specimens of submucosal gastric cancers, but 8 were excluded because of the following reasons: i) the part with submucosal invasion was unclear on immunohistochemistry due to inappropriate technique for preparing the pathological sections; ii) the depth of submucosal invasion was unclear due to problems during the endoscopic procedure; and iii) cancers with ulceration or ulcer scars because of difficulties identifying the muscularis mucosae. Finally, 44 specimens were considered eligible and analyzed in this study.

All specimens had been fixed in $10 \%$ buffered neutral formalin, embedded in paraffin, and cross-cut into 2 - $\mu \mathrm{m}$-thick sections. Hematoxylin and eosin (HE)-stained sections were prepared and observed under a microscope, and the sections in which cancer cells reached the deepest level of the submucosa were subjected to detailed examination. Histological classification was performed by two independent board-certified pathologists in our hospital according to the Japanese Classification of Gastric Carcinoma. When a disagreement arose, it was discussed until a consensus was reached. The phenotypic differences between the mucosal and submucosal components were investigated, along with the patterns of tumor invasion to the submucosal layer.

The study protocol was approved by the ethics committee of Wakayama Medical University. Informed consent was obtained from each patient.

\section{Immunohistochemistry}

MUC5AC and MUC6 were used as gastric phenotypic markers, while MUC2 and CD10 were used as intestinal phenotypic markers. The MUC5AC glycoprotein is known to react with epithelial cells in the surface of gastric foveola. The MUC6 glycoprotein is expressed in mucous cells of the neck zone of oxyntic mucosa and in pyloric gland cells [17]. The MUC2 glycoprotein is expressed in the supranuclear area of goblet cells in mucosal areas showing intestinal metaplasia in the stomach $[18,19]$. The CD10 glycoprotein is expressed on the brush border of intestinal epithelial cells [20,21]. For immunohistochemistry, the following monoclonal antibodies were used as primary antibodies: MUC5AC (1:40, CLH2; Novocastra, Newcastle, UK), MUC6 (1:100, CLH5; Novocastra), MUC2 (1:40, Ccp58; Novocastra), and CD10 (1:200000, 56C6; DAKO, Glostrup, Denmark). With regard to the epithelial cell markers, the results of immunohistochemical staining were evaluated in terms of the percentage of positive cytoplasmic and luminal stained cancer cells, with $10 \%$ and above considered positive.

The immunohistochemical expression of Ki-67 antigen was also examined using the MIB1 monoclonal antibody (1:200, MIB-1, DAKO) to assess the aggressiveness of the gastric carcinomas studied. Any nuclear staining, regardless of the intensity of the reaction, was considered positive for Ki-67. The reaction was quantified through the assessment of a marking index of Ki-67 (MI Ki-67), expressed as a percentage result of the number of Ki-67+ cells reported of 500 cells (Ki-67+ and Ki-67-). MI Ki-67 was evaluated in the submucosal component of the tumor.

\section{Classification of phenotypic expression of carcinomas}

Tumors were classified phenotypically with reference to the expression patterns of a battery of epithelial cell markers. Mucin phenotype expression was judged as follows: i) Gastric phenotype (Gtype): MUC5AC- and/or MUC6-positive rate of $10 \%$ or more; ii) Intestinal phenotype (I-type): MUC2- and/or CD10-positive rate of $10 \%$ or more; iii) Gastric-and-intestinal mixed phenotype (GI-type): MUC5AC- and/or MUC6-positive as well as MUC2- and/or CD10positive rates of $10 \%$ or more; and iv) Null phenotype (N-type): Positive rates for all the four markers less than $10 \%$. The phenotypic expressions of the mucosal and submucosal layers were evaluated individually based on the above classification.

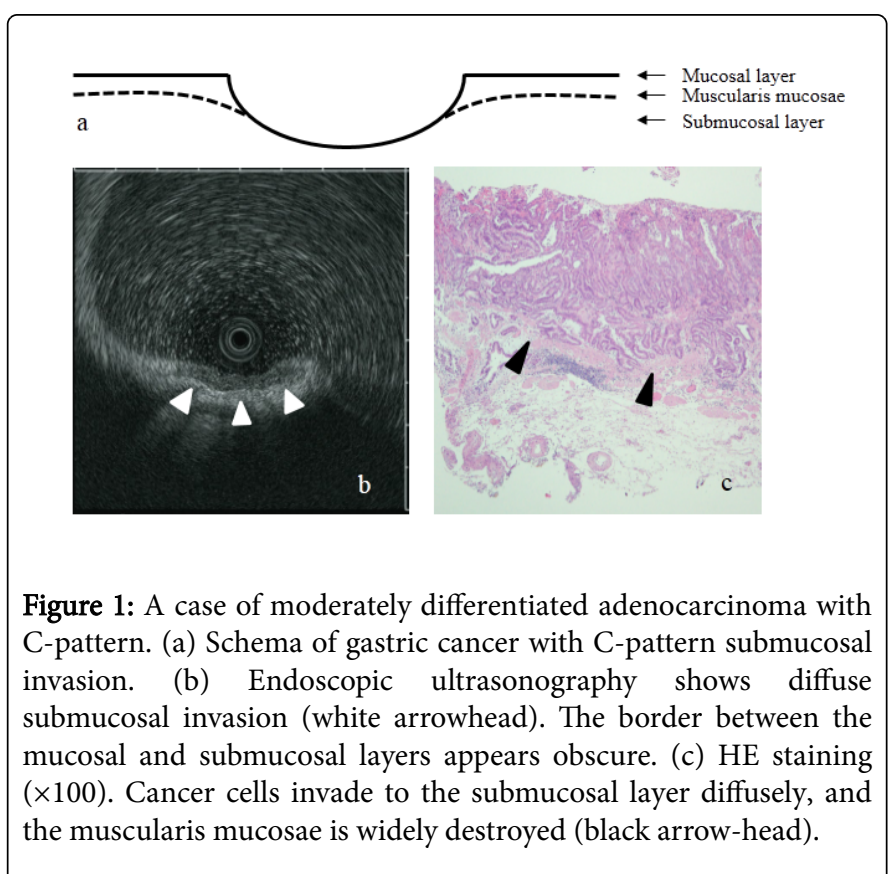


Citation: Fukatsu K, Kato J, Niwa T, Iguchi M, Muraki Y, et al. (2015) Microscopic Invasion Patterns and Epithelial Cell-phenotypes in Early Gastric Cancer with Submucosal Invasion. J Cytol Histol 6: 380. doi:10.4172/2157-7099.1000380

Page 3 of 6

\section{Patterns of submucosal invasion}

The pattern of tumor invasion to the submucosal layer was classified into two categories: collapsing pattern (C-pattern) and passingthrough pattern (P-pattern). C-pattern was defined as a pattern in which cancer cells invaded to the submucosal layer with expansive destruction of a wide range of muscularis mucosae. In cancers with Cpattern, the muscularis mucosae beneath the cancer was pressed down, and the histological border between the mucosal and submucosal layers appeared obscure (Figures 1a-1c). In contrast, in P-pattern invasion, the cancer cells penetrate the muscularis mucosae and form focal cancer cell aggregations in the submucosal layer, with little or no destruction of the muscularis mucosae. The histological structure of the muscularis mucosae is spared except for the site of penetration, and cancer cells form a neck-like appearance between the mucosal and submucosal layers (Figures 2a-2c).

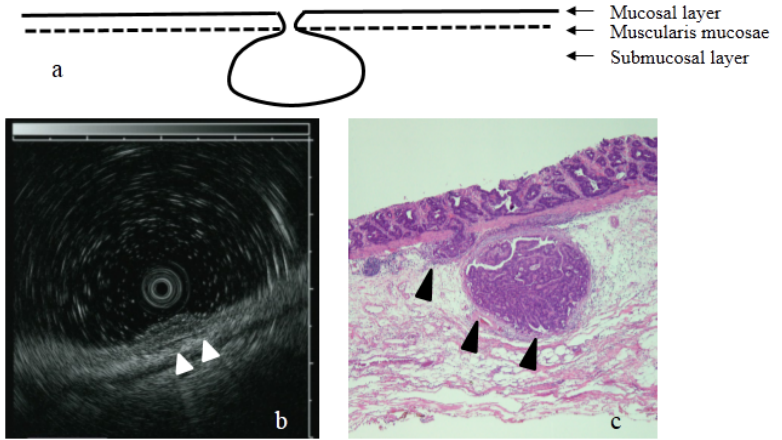

Figure 2: A case of moderately differentiated adenocarcinoma with P-pattern. (a) Schema of gastric cancer with P-pattern submucosal invasion. (b) Endoscopic ultrasonography shows hypoechoic lesions in the third layer, which indicate focal submucosal invasion (white arrowhead). (c) HE staining $(\times 100)$. Cancer cells focally invade to the submucosal layer like passing through the muscularis mucosae, which is maintained (black arrow-head).

\section{Statistical analyses}

Statistical analyses were performed using the $\chi^{2}$ test, Fisher's exact test, and the Mann-Whitney $U$ test as appropriate. The values were considered significantly different when the $\mathrm{p}$ value was less than 0.05 . SPSS version 11.0 (Chicago, IL) was used for all calculations.

\section{Results}

Of the 44 analyzed submucosal gastric cancers, 19 were categorized as C-pattern, while 25 were P-pattern. Table 1 shows the clinicopathological features of the cancers in each pattern. Most clinicopathological features showed no differences between the two patterns of cancers. In terms of tumor location, however, P-pattern cancers were more frequent in the upper or middle portion of the stomach. Cancers with deep (more than $500 \mu \mathrm{m}$ ) submucosal invasion and/or with vessel invasion were similarly observed in both groups, and one P-pattern case was found to have lymphatic metastases with additional surgery. Epithelial cell phenotypes in the mucosal layer of C- pattern and P-pattern tumors were 4 G-type, 6 GI-type, and 9 I-type, and 3 G-type, 11 GI-type, and 11 I-type, respectively.

\begin{tabular}{|l|l|l|l|}
\hline & $\begin{array}{l}\text { C-pattern } \\
(\mathbf{n = 1 9 )}\end{array}$ & $\begin{array}{l}\text { P-pattern } \\
\text { (n=25) }\end{array}$ & P value \\
\hline Age, median (range), y & $68(58-90)$ & $67(54-84)$ & 0.38 \\
\hline Sex (Male/Female) & $17 / 2$ & $24 / 1$ & 0.4 \\
\hline Location (Upper or Middle/Lower) & $11-$ Aug & $18 / 7$ & 0.04 \\
\hline Size, median (range), mm & $17(8-60)$ & $17(7-55)$ & 0.23 \\
\hline Macroscopic type (elevated, depressed) & $10-\mathrm{Sep}$ & $17-\mathrm{Aug}$ & 0.3 \\
\hline Histological type (tub1,tub2,pap) & $11 / 6 / 2002$ & $16 / 9 / 0$ & 0.25 \\
\hline Lymphatic or venous invasion (+/-) & $13-\mathrm{Jun}$ & $18-\mathrm{Jul}$ & 0.79 \\
\hline Depth of invasion (sm1/sm2) & $9-\mathrm{Oct}$ & $15-\mathrm{Oct}$ & 0.41 \\
\hline MI Ki-67, median (range) & $62(46-72)$ & $58(44-76)$ & 0.47 \\
\hline Phenotype of mucosal layer (G/GI/I) & $4 / 6 / 2009$ & $3 / 11 / 2011$ & 0.6 \\
\hline
\end{tabular}

Upper and middle, upper and middle thirds of the stomach; Lower, lower third of the stomach; tub1, well differentiated tubular adenocarcinoma; tub2, moderately differentiated tubular adenocarcinoma; pap, papillary adenocarcinoma; sm1, submucosal invasion of less than $500 \mathrm{~mm}$; sm2, submucosal invasion of 500 $\mathrm{mm}$ or more.

Table 1: Clinicopathological features of analyzed gastric cancers.

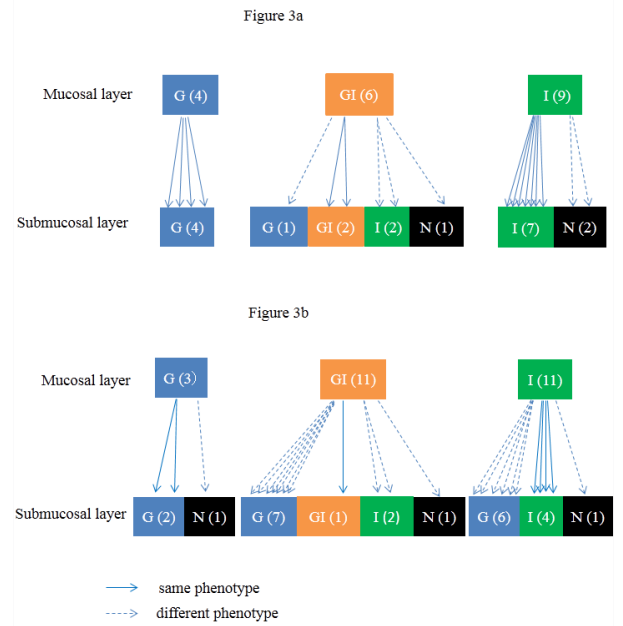

Figure 3: Epithelial cell-phenotype differences between the mucosa and submucosa. Cancers with C-pattern invasion are likely to show similar phenotypes between the mucosa and submucosa, while phenotypes of cancers with P-pattern invasion are likely to differ between the two layers (rate of the same phenotypes: C-pattern 68\% vs. P-pattern $28 \%, \mathrm{p}=0.008$ ). In the submucosal layer, in particular, G-type is more frequently observed in cancers with P-pattern invasion than in those with C-pattern invasion $(60 \%$ vs. $26 \%, p=$ 0.026). (a) C-pattern. (b) P-pattern. 
The phenotypic differences between the mucosal and submucosal components in each cancer by invasion pattern are shown in Figure 3. Cancers with C-pattern invasion were likely to show similar phenotypes between the mucosa and submucosa, while phenotypes of cancers with P-pattern invasion were likely to be different between the two layers (rate of the same phenotypes: C-pattern $68 \%$ vs. P-pattern $28 \%, \mathrm{p}=0.008)$. In the submucosal layer, in particular, G-type was more frequently observed in cancers with $\mathrm{P}$-pattern invasion than in those with C-pattern invasion ( $60 \%$ vs. $26 \%, p=0.026$ ). Of 22 cancers with P-pattern invasion with an intestinal phenotype component in the mucosa, $15(68 \%)$ lost the intestinal phenotype component in the submucosa, and 13 (59\%) showed G-type alone in the submucosa. In contrast, of $15 \mathrm{C}$-pattern cancers with an intestinal phenotype component, only 1 (6.7\%) showed G-type alone in the submucosa. Null type was seen in the submucosal layer alone in each invasion group. These results suggest that the phenotype presentation in the mucosa and submucosa differs according to invasion pattern, and that tumors with P-pattern invasion are likely to express the gastric phenotype in the submucosa regardless of the phenotype in the mucosa.
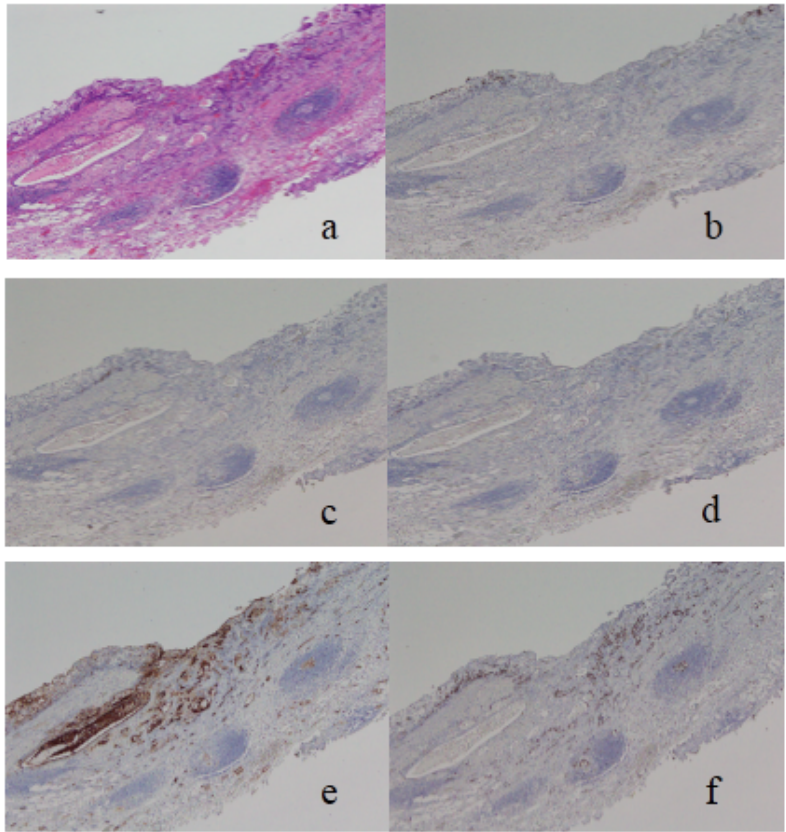

Figure 4: Histology and immunohistochemistry of gastric cancer with C-pattern submucosal invasion. A case of moderately differentiated adenocarcinoma of type 0-IIc, measuring $12 \mathrm{~mm}$ in diameter, with submucosal invasion of $>500 \mu \mathrm{m}$ is shown. Both mucosal and submucosal layers are I-type. (a) HE staining $(\times 100)$ (b) MUC5AC-negative $(\times 100)$ (c) MUC6-negative $(\times 100)$ (d) MUC2-negative $(\times 100$ (e) CD10-positive both in the mucosal and submucosal layers $(\times 100)$ (f) MI Ki-67 is $61 \%$.

Figures 4 and 5 show HE staining and immunohistochemical expressions of C-pattern and P-pattern cancers, respectively. In Figure 4 , tumor cells in both the mucosal and submucosal layers were positive for CD10 and negative for MUC2, MUC5AC, and MUC6, indicating that the tumor was classified as I-type both in the mucosa and in the submucosa. In Figure 5, the phenotype of the mucosal layer was I-type with positive CD10 expression and with negative expressions of MUC2, MUC5AC, and MUC6. In contrast, the phenotype of the submucosal layer was G-type with positive expression of MUC5AC alone.

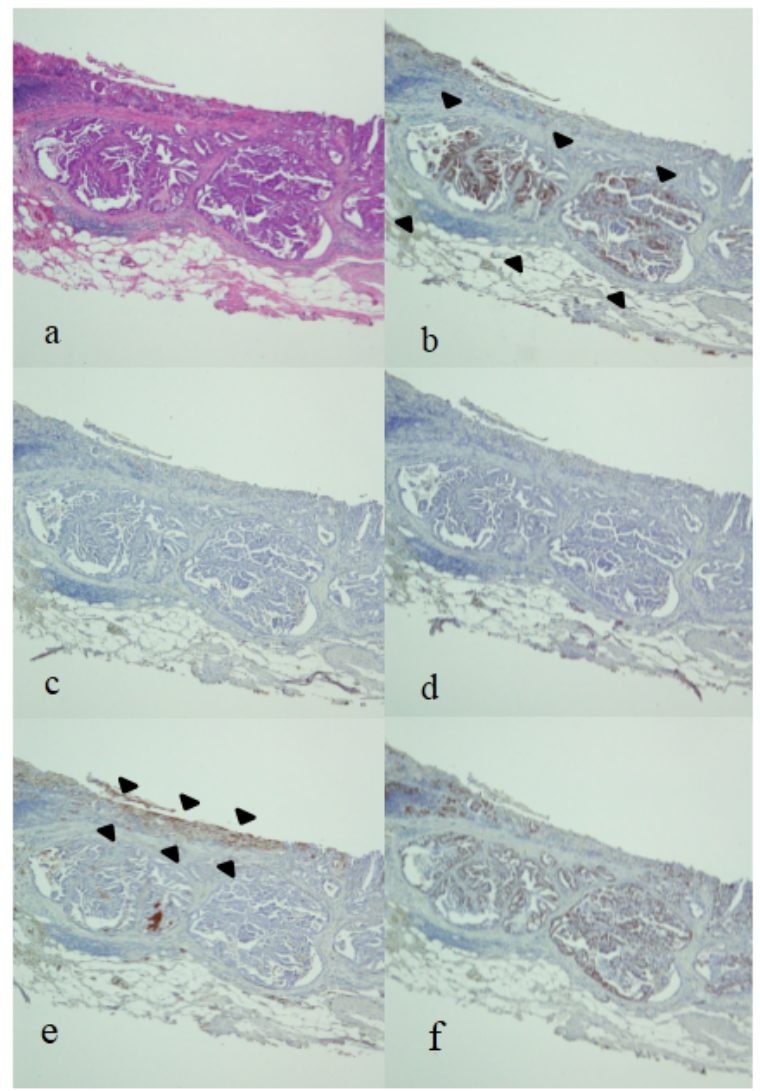

Figure 5: Histology and immunohistochemistry of gastric cancer with P-pattern submucosal invasion. A case of moderately differentiated adenocarcinoma of type 0 -IIc, measuring $8 \mathrm{~mm}$ in diameter, with submucosal invasion of $>500 \mu \mathrm{m}$. The mucosal layer is I-type, while the submucosal layer is G-type. (a) HE staining $(\times 100)$. (b) MUC5AC-positive only in the submucosal layer (black arrow-head $)(\times 100)$. (c) MUC6-negative $(\times 100)$. (d) MUC2negative $(\times 100)$. (e) CD10-positive only in the mucosal layer (black arrow-head) ( $\times 100)$. (f) MI Ki-67 is $70 \%$.

\section{Discussion}

In the present study, the difference in the phenotypic expression between the mucosal and submucosal layers of early gastric cancer was evaluated with reference to the pattern of tumor invasion to the submucosal layer. In cancers showing C-pattern invasion, the phenotypes of the mucosa and submucosa are likely to be similar, while in cancers with P-pattern invasion, the phenotypes are likely to differ between the two layers. In particular, cancer cells in the submucosa of the tumor with P-pattern invasion are more likely to be G-type, and the I-type component in the mucosa of the tumors was frequently lost in the submucosa. The specific correlations between 
invasion pattern and phenotype presentation suggest the presence of unique mechanisms of gastric cancer progression.

Most gastric cancers, independent of the histological type, are considered to occur with a complete gastric phenotype in the early stage and acquire intestinal phenotypic expression in the proliferating zone as they grow. Components of the intestinal phenotype then spread outside the proliferating zone and the whole tumor shifts to an intestinal phenotype [9-12]. Intestinalization is at least partly caused by Helicobacter pylori (H. pylori) infection, which is strongly associated with intestinal-specific gene expression, such as Caudal-related homeobox gene (Cdx) 1 and $\mathrm{Cdx} 2$ [22-24]. In this context, Yamamoto et al. reported that approximately $70 \%$ of gastric cancers found after eradication of $\mathrm{H}$. pylori were complete gastric type or gastricpredominant mixed type $[25,26]$. In addition, Kato et al. reported that most $\mathrm{H}$. pylori infection-negative gastric cancers were complete gastric type or gastric-predominant mixed type [27].

In terms of $H$. pylori infection, the following explanations may be plausible for our findings. In P-pattern invasion, the tumor cells pass through the space of the muscularis mucosae and invade to the submucosal layer at an early stage while the intestinalization does not yet occur. Thereafter, they grow individually in the mucosal and submucosal layers. Tumor cells in the mucosal layer acquire intestinal phenotype by exposure to $H$. pylori, whereas those in the submucosal layer maintain the gastric phenotype because of the lack of $H$. pylori and presumably related factors. The alternative scenario is the reversion to the gastric phenotype from the intestinal phenotype along with invasion to the submucosa possibly due to the decrease of factors correlated with intestinalization present only in the mucosa. On the other hand, in C-pattern invasion, the tumor cells destroy the muscularis mucosae and invade to the submucosal layer diffusely. During such a process, tumor cell phenotype is unlikely to change, because factors that determine the phenotypes also appear to infiltrate to the submucosa.

In the present study, cancers with P-pattern invasion were more frequently located in the middle or upper portion of the stomach. This result may be attributed to the difference in the structure of the muscularis mucosae among gastric portions. It is known that vessels penetrating the muscularis mucosae are more frequently observed in the upper or middle portion of the stomach than in the lower portion. In addition, the thickness of the muscularis mucosae of the middle or upper body is thinner than that of the lower body. Thus, the thin and gappy structure of the muscularis mucosae may facilitate P-pattern submucosal invasion. The present study cohort included more Ppattern than C-pattern cancers, although the depth of invasion had been fully evaluated in all cases prior to treatment, and the diagnosis of submucosal cancer usually prohibited endoscopic resection. This suggests that precise diagnosis of P-pattern submucosal invasion prior to resection is difficult. Therefore, the indication for endoscopic resection of cancer in the upper or middle portion of the stomach should be carefully determined.

Nakamura et al. previously reported that there were no combinations of a gastric phenotype in the mucosal layer and an intestinal phenotype in the submucosal layer, or an intestinal phenotype in the mucosal layer and a gastric phenotype in the submucosal layer in surgically resected gastric cancer with submucosal invasion [28]. Their findings were not consistent with the present findings regarding the latter, in particular, in cancers with P-pattern invasion. This difference may be attributed to the depth of cancer invasion. Surgically resected cancer is considered to harbor deeper submucosal invasion than endoscopically resected submucosal cancer. Cancers showing P-pattern submucosal invasion with I-type in the mucosa and G-type in the submucosa might lose the gastric phenotype component of the submucosa along with the deeper advance of submucosal invasion, because, even in cancers showing the P-pattern, massive submucosal invasion would destroy the muscularis mucosae and factors associated with intestinalization would penetrate into the submucosa.

It has been reported that differentiated adenocarcinoma with gastric phenotype has a high malignant potential [29-31], and Koseki et al. reported that cancer with the gastric phenotype is significantly more likely to invade to vessels or metastasize to lymph nodes than that with the intestinal phenotype or mixed type [32]. Cancer with P-pattern invasion, therefore, may have high malignant potential because G-type is more frequently observed in the deepest region of those cancers. In the present study, in fact, one case of submucosal invasive cancer with lymphatic metastasis showed P-pattern invasion, and cells in the submucosal layer of the case showed a completely gastric phenotype. However, the difference in prognosis between cancers with the gastric and intestinal phenotypes is controversial [33], and the Ki-67 index was not significantly different between the two patterns of cancers in the present study. Further studies are needed to clarify the malignant potential of gastric cancer according to cell phenotypes and patterns of submucosal invasion.

Null type was seen only in the submucosal layer in the present study. The result is consistent with that of the previous report in which the authors indicated that loss of phenotypic expression occurred during the course of invading the submucosa or creating metastases, and that the loss was associated with dedifferentiation of cancer histology [28]. The null type cancer seen in the present study, therefore, might advance into undifferentiated cancer during progression.

This study has limitations. In particular, the number of analyzed cancers was relatively small, and all were differentiated and endoscopically resected cancers. Analysis of a larger number of cancers including other histological types and various depths of invasion may reveal more detailed correlations between gastric cancer invasion and epithelial cell phenotypes. It is also known that E-cadherin and Matrix metalloproteinases (MMPs) are associated with tumor invasion and metastasis in gastric cancer [34,35], and further studies are needed in order to examine the relationship between such factors and invasion pattens.

In conclusion, the results of the present study showed that cancer cell phenotype presentations in the mucosa and submucosa are correlated with the patterns of tumor invasion into the submucosal layer in early gastric cancer. Cancers with P-pattern invasion are likely to express the gastric phenotype in the submucosa even when the phenotype of the mucosa showed the intestinal type, suggesting the presence of unique invasion mechanisms in this type of cancers. The present findings may help, at least in part, to elucidate the mechanisms of gastric cancer invasion.

\section{References}

1. Ohtsuka H, Nagamatsu S (2006) Changing trends in the number of deaths from soft tissue sarcoma in Japan, 1955-2002. Eur J Cancer Care (Engl) 15: 133-137.

2. Sano T, Kobori O, Muto T (1992) Lymph node metastasis from early gastric cancer: endoscopic resection of tumour. Br J Surg 79: 241-244. 
3. Kadokura H (1968) A study on the extent and intramural growth of carcinoma of stomach. J Jpn Surg Soc 69: 555-564.

4. Inokuchi K, Soejima K (1976) The clinical consideration on the progress of the early gastric cancer analyzed from its growth pattern. Surgical Therapy 34: 49-54.

5. Lauren P (1965) The two histological main types of gastric carcinoma: diffuse and so-called intestinal-type carcinoma. an attempt at a histoclinical classification. Acta Pathol Microbiol Scand 64: 31-49.

6. Sugano H, Nakamura K, Kato Y (1982) Pathological studies of human gastric cancer. Acta Pathol Jpn 32 Suppl 2: 329-347.

7. Ming SC (1977) Gastric carcinoma. A pathobiological classification. Cancer 39: 2475-2485.

8. Correa P (1988) A human model of gastric carcinogenesis. Cancer Res 48: 3554-3560.

9. Watanabe H (1996) The pathological sequence in the development of gastric cancer: II. Scand J Gastroenterol Suppl 214: 36-39.

10. Egashira Y, Simoda T, Ikegami M (1999) Mucin histochemical analysis of minute gastric differentiated adenocarcinoma. Pathol Int 49: 55-61.

11. Yamachika T, Inada K, Fujimitsu Y, Nakamura S, Yamamura Y, et al. (1997) Intestinalization of gastric signet ring cell carcinomas with progression. Virchows Arch 431: 103-110.

12. Yoshikawa A, Inada K, Yamachika T, Shimizu N, Kaminishi M, et al. (1998) Phenotypic shift in human differentiated gastric cancers from gastric to intestinal epithelial cell type during disease progression. Gastric Cancer 1: 134-41.

13. Tatematsu M, Tsukamoto T, Inada $\mathrm{K}$ (2003) Stem cells and gastric cancer: role of gastric and intestinal mixed intestinal metaplasia. Cancer Sci 94: 135-141.

14. Tatematsu M, Tsukamoto T, Mizoshita T (2005) Role of Helicobacter pylori in gastric carcinogenesis: the origin of gastric cancers and heterotopic proliferative glands in Mongolian gerbils. Helicobacter 10: 97-106.

15. Mizoshita T, Tsukamoto T, Toyoda T, Ban H, Nozaki K, et al. (2007) Intestinal phenotypes of stomach cancers arising after Helicobacter pylori eradication In carcinogen-treated Mongolian gerbils. Asian Pac J Cancer Prev 8: 267-271.

16. Japanese Research Society for Gastric Cancer (1995). Japanese Classification of Gastric Carcinoma. 1st English edn. Tokyo: Kanehara.

17. Reis CA, David L, Correa P, Carneiro F, de Bolos C, et al. (1999) Intestinal metaplasia of human stomach displays distinct patterns of mucin (MUC, MUC, MUC5AC, and MUC6) expression. Cancer Res 59: 1003-7.

18. Ajioka Y, Watanabe H, Jass JR (1997) MUC1 and MUC2 mucins in flat and polypoid colorectal adenomas. J Clin Pathol 50: 417-421.

19. Tytgat KM, Büller HA, Opdam FJ, Kim YS, Einerhand AW, et al. (1994) Biosynthesis of human colonic mucin: Muc2 is the prominent secretory mucin. Gastroenterology 107: 1352-1363.

20. Endoh Y, Tamura G, Motoyama T, Ajioka Y, Watanabe H (1999) Welldifferentiated adenocarcinoma mimicking complete-type intestinal metaplasia in the stomach. Hum Pathol 30: 826-32.

21. Sato Y, Itoh F, Hinoda Y, Ohe Y, Nakagawa N, et al. (1996) Expression of $\mathrm{CD} 10 /$ neutral endopeptidase in normal and malignant tissues of the human stomach and colon. J Gastroenterol 31: 12-17.
22. Mizoshita T, Tsukamoto T, Takenaka Y, Cao X, Kato S, et al. (2006) Gastric and intestinal phenotypes and histogenesis of advanced glandular stomach cancers in carcinogen-treated, Helicobacter pylori-infected Mongolian gerbils. Cancer Sci 97: 38-44.

23. Eda A, Osawa H, Yanaka I, Satoh K, Mutoh H, et al. (2002) Expression of homeobox gene $\mathrm{CDX} 2$ precedes that of $\mathrm{CDX} 1$ during the progression of intestinal metaplasia. J Gastroenterol 37: 94-100.

24. Mizoshita T, Inada K, Tsukamoto T, Kodera Y, Yamamura Y, et al. (2001) Expression of Cdx 1 and $\mathrm{Cdx} 2 \mathrm{mRNAs}$ and relevance of this expression to differentiation in human gastrointestinal mucosa-with special emphasis on participation in intestinal metaplasia of the human stomach. Gastric Cancer 4: 185-191.

25. Yamamoto K, Kato M, Takahashi M, Haneda M, Shinada K, et al. (2011) Clinicopathological analysis of early -stage gastric cancers detected after successful eradication of Helicobacter pylori. Helicobacter 16: 210-216.

26. Matsuo T, Ito M, Tatsugami M, Boda T, Takata S, et al. (2012) Gastric cancer development after Helicobacter pylori eradication therapy: a new form of gastric neoplasia. Digestion 85: 61-67.

27. Kato S, Matsukura N, Tsukada K, Matsuda N, Mizoshita T, et al. (2007) Helicobacter pylori infection-negative gastric cancer in Japanese hospital patients: Incidence and pathological characteristics. Cancer Sci 98: 790-794.

28. Nakamura T, Yao T, Kabashima A, Nishiyama K, Maehara Y, et al. (2005) Loss of phenotypic expression is related to tumour progression in early gastric differentiated adenocarcinoma. Histopathology 47: 357-367.

29. Kabashima A, Yao T, Sugimachi K, Tsuneyoshi M (2002) Relationship between biologic behavior and phenotypic expression in intramucosal gastric carcinomas. Hum Pathol 33: 80-86.

30. Shibata N, Watari J, Fujiya M, Tanno S, Saitoh Y, et al. (2003) Cell kinetics and genetic instabilities in differentiated type early gastric cancers with different mucin phenotype. Hum Pathol 34: 32-40.

31. Tajima Y, Shimoda T, Nakanishi Y, Yokoyama N, Tanaka T, et al. (2001) Gastric and intestinal phenotypic marker expression in gastric carcinomas and its prognostic significance: immunohistochemical analysis of 136 lesions. Oncology 61: 212-20.

32. Koseki K, Takizawa T, Koike M, Ito M, Nihei Z, et al. (2000) Distinction of differentiated type early gastric carcinoma with gastric type mucin expression. Cancer 89: 724-732.

33. Wakatsuki K, Yamada Y, Narikiyo M, Ueno M, Takayama T, et al. (2008) Clinicopathological and prognostic significance of mucin phenotype in gastric cancer. J Surg Oncol 98: 124-129.

34. Oka H, Shiozaki H, Kobayashi K, Tahara H, Tamura S, et al. (1992) Immunohistochemical evaluation of E-cadherin adhesion molecule expression in human gastric cancer. Virchows Arch A Pathol Anat Histopathol 421: 149-156.

35. Zheng H, Takahashi H, Murai Y, Cui Z, Nomoto K, et al. (2006) Expressions of MMP-, MMP-9 and VEGF are closely linked to growth, invasion, metastasis and angiogenesis of gastric carcinoma. Anticancer Res 26: 3579-83. 